\title{
Projeto RESPIRE: protocolo de avaliação do impacto da pandemia na saúde da comunidade do IFSul
}

\section{RESPIRE Project: protocol for evaluating the impact of the pandemic on the health of the IFSul community}

\section{AUTORES \\ Cesar Augusto Häfele ${ }^{1}$ (D) \\ Marina Marques Kremer ${ }^{2,3}$ (D) \\ Gabriel Barros da Cunha ${ }^{2,3}$ (D) \\ Gicele de Oliveira Karini da Cunha $a^{2,3}$ (D) \\ Italo Fontoura Guimarães ${ }^{1,3}$ (D) \\ Fabricio Mota Foster ${ }^{3}$ iD \\ Giancarlo Bacchieri ${ }^{2,3}$ (D) \\ Felipe Fossati Reichert ${ }^{1}$ (D) \\ Thiago Terra Borges ${ }^{2,3}$ (D) \\ 1 Universidade Federal de Pelotas, Departamento de Educação Física, Pelotas, Rio Grande do Sul, Brasil. \\ 2 Instituto Federal Sul-rio-grandense, \\ Departamento de Educação Física, Câmpus Pelotas, Pelotas, Rio Grande Sul, Brasil. \\ 3 Instituto Federal de Sul-rio-grandense, \\ Especialização em Esporte Escolar, Câmpus Pelotas, Pelotas, Rio Grande Sul, Brasil.}

\section{CONTATO}

Cesar Augusto Häfele

hafele.c@hotmail.com

Rua Praça 20 de setembro, n. 455. Pelotas,

Rio Grande do Sul, Brasil.

CEP: 96015-360.

DOI

$10.12820 /$ rbafs. $27 \mathrm{e} 0244$

\section{(cc) BY}

Este trabalho está licenciado com uma Licença Creative Commons - Atribuição 4.0 Internacional.

\begin{abstract}
RESUMO
A pandemia do coronavírus (COVID-19) configura-se como uma das maiores crises sanitárias já enfrentadas pela humanidade. Este protocolo detalha o estudo sobre o impacto do distanciamento social na saúde de escolares e servidores dos 14 Câmpus e Reitoria do Instituto Federal Sul-rio-grandense (IFSul). O estudo observacional longitudinal envolve duas coletas de dados: Coleta 1 - realizada entre os meses de maio e agosto de 2021 com informações referentes ao período pré e durante o distanciamento social; Coleta 2 - será realizada no retorno das aulas presenciais no período pós distanciamento social (previsto para abril de 2022). Participantes responderam um questionário auto aplicado através da plataforma QuestionPro, onde foram coletadas variáveis sociodemográficas, comportamentais (atividades físicas, sedentárias, sono, alimentação, tabagismo e consumo de álcool), saúde mental, autopercepção de saúde e relacionadas a instituição de ensino. Estratégias de alcance da população alvo envolveram a utilização da página do Câmpus, aplicação do questionário em aula para alunos, encaminhamento via e-mail aos servidores e divulgação nas mídias sociais. Entender como o distanciamento social afetou a saúde de estudantes e servidores e realizar um acompanhamento no retorno das aulas é importante para os gestores terem um diagnóstico de saúde dos diferentes momentos, criarem políticas para facilitar o retorno as aulas, bem como, auxiliar no enfrentamento dos problemas identificados com a pesquisa durante o retorno as aulas presenciais.
\end{abstract}

Palavras-chave: Saúde do estudante; Saúde do trabalhador; Escola; Protocolo.

\section{ABSTRACT}

The coronavirus pandemic (COVID-19) is one of the biggest health crises ever faced by humanity. This protocol details the study on the impact of social distancing on the health of students and employees of the 14 college and Rectory of the Federal Institute of Sul-rio-grandense (IFSul). The longitudinal observational study involves two data collections: Collect 1 - carried out between May and August 2021 with information regarding the period before and during social distancing; Collect 2 - will be carried out on the return of in-person classes in the post-social distancing period (Scheduled for April 2022). Participants answered a self-administered questionnaire through the QuestionPro platform, where sociodemographic, behavioral (physical and sedentary activities, sleep, diet, smoking and alcohol consumption), mental health, self-perceived health and educational institution-related variables were collected. Strategies to reach the target population involved using the Campus page, applying the questionnaire in class to students, forwarding it via email to servers and dissemination on social media. Understanding how social distancing has affected the health of students and employees and monitoring the return to classes is important for managers to have a health diagnosis of the different moments, create policies to facilitate the return to classes, as well as help in coping with problems identified with the research during the return to in-person classes.

Keywords: Student health; Occupational health; Schools; Protocols.

\section{Introdução}

A pandemia ocasionada pelo novo coronavírus (COVID-19) constitui-se como uma das maiores crises sanitárias já enfrentadas pela humanidade. Mesmo após mais de um ano de esforços científicos, políticos e econômicos, muitos países, em diferentes regiões do planeta, enfrentam dificuldade em conter o avanço do vírus SARS-CoV-2. Em junho de 2021, cerca de 181 milhões de pessoas no mundo já haviam sido infectadas, ocasionando aproximadamente 4 milhões de óbitos. No Brasil, epicentro da doença em junho de 2021, os números são alarmantes. Ao ultrapassar a marca de meio milhão de óbitos, o Brasil já representava mais de 13\% das mortes por COVID-19 no mundo ${ }^{1}$.

Inserido neste cenário caótico de pandemia, o Rio Grande do Sul, estado localizado mais ao sul do Bra- 
sil, tem adotado medidas preventivas que visam reduzir o número de pessoas infectadas e minimizar o impacto no sistema de saúde. Além da recomendação do uso de máscaras e higienização das mãos, o distanciamento social tem sido fortemente sugerido pelos órgãos de saúde como forma de conter o avanço da doença $\mathrm{a}^{2-4}$. No entanto, a adoção de um modelo de distanciamento social provoca alterações significativas no cotidiano da população, acarretando, em muitos casos, a redução no convívio social, o fechamento de parques e praias, estabelecimentos comerciais e instituições de ensino. Esse modelo de distanciamento social, embora muitas vezes necessário, pode ocasionar mudanças no estilo de vida da população ${ }^{5}$.

Mesmo que as consequências do distanciamento social acometam toda a população, há uma preocupação com os possíveis efeitos na saúde física e mental da comunidade escolar ${ }^{6}$. Sendo a escola um espaço de desenvolvimento cognitivo, afetivo, social, emocional e psicomotor, as políticas de reclusão impostas pelo distanciamento social podem tornar-se um problema de natureza holística ${ }^{7,8}$. A diminuição das atividades realizadas ao ar livre e a redução na interação social entre os escolares durante o período de ensino remoto podem acarretar graves consequências na saúde e no desenvolvimento de crianças e adolescentes ${ }^{6,9}$.

Estudos recentes têm mostrado que durante operíodo de distanciamento social os escolares diminuíram a prática de atividades físicas ${ }^{10,11} \mathrm{e}$ aumentaram o tempo de exposição a telas ${ }^{10,12}$. Esse panorama tem se revelado nocivo as crianças e adolescentes, aumentando os problemas de excesso de peso, transtornos alimentares, depressão, ansiedade e distúrbios do sono ${ }^{2,5,6,11,13}$. Solon Jr e colaboradores ${ }^{14}$ destacam a importância da manutenção da prática de atividade física neste cenário. Os pesquisadores identificaram maior percepção de qualidade de vida, menores índices de ansiedade e maior qualidade no sono em sujeitos ativos no período de distanciamento social quando comparados aqueles que eram fisicamente inativos no mesmo período ${ }^{14}$.

No que tange aos professores, além dos efeitos gerais da pandemia, o ensino no cenário remoto obrigou-os a uma reinvenção da prática pedagógica em caráter emergencial ${ }^{8}$. Dentro desse contexto, é importante destacar que a atividade docente passa por um momento de sensível reformulação, visto que a atuação em cenário remoto promove modificações nas características basilares do processo educativo ${ }^{15}$. Ademais, a mudança no cenário de trabalho docente pode representar não apenas um aumento na carga de trabalho ${ }^{16}$, como também a necessidade de investir mais tempo em processos de qualificação para aperfeiçoar o uso de tecnologias digitais que permitam maior adequação ao contexto das aulas ${ }^{15}$.

Os servidores técnico-administrativos em educação (TAE) também foram acometidos por alterações na rotina de trabalho. Por tratar-se de um cargo de natureza eclética, diferentes ajustes precisaram ser feitos para atender a demandas específicas de cada atividade nos mais diversos setores. Apesar de ainda serem escassos os estudos que buscam identificar o impacto do distanciamento social na atividade laboral dos TAEs, é provável que a modificação na dinâmica de trabalho do modo presencial para o remoto possa ter acarretado em novos desafios para a manutenção do volume e qualidade de trabalho.

Diversos estudos, inclusive de base populacional, foram realizados com o objetivo de identificar o impacto do distanciamento social nos indicadores de saúde de $\operatorname{adultos}^{17}$, adolescentes e crianças $^{18}$. Esses avanços científicos têm permitido identificar possíveis efeitos do distanciamento social na saúde física e mental da população. No entanto, a escola é um espaço social que sofreu profundas alterações na sua dinâmica de funcionamento, merecendo assim, maior atenção.

Pesquisas que se proponham a estudar o contexto escolar poderão auxiliar em estratégias de suporte a saúde que atendam a possíveis demandas específicas dos estudantes e profissionais da educação. Além disso, poucos são os estudos que se propuseram a investigar de forma longitudinal os impactos do distanciamento social sobre o nível de atividade física e suas relações com outros indicadores de saúde. Por fim, outra contribuição do presente estudo será a proposição de uma estrutura de investigação possível de ser replicada em outras instituições de ensino.

Diante do exposto, a pesquisa RESPIRE, tem por objetivo verificar o impacto do distanciamento social imposto pela pandemia da COVID-19 nas relações entre a atividade física e os indicadores de saúde dos estudantes e servidores do Instituto Federal de Educação Ciência e Tecnologia Sul-rio-grandense (IFSul). $\mathrm{O}$ estudo permitirá verificar não apenas o impacto do distanciamento social na vida de estudantes e servidores, mas também os possíveis efeitos do retorno às aulas presenciais.

\section{Métodos}

A RESPIRE é um estudo observacional longitudinal 
ambidirecional do tipo Censo no Instituto Federal de Educação, Ciência e Tecnologia Sul-rio-grandense (IFSul). O protocolo do estudo foi aprovado pelo $\mathrm{Co}_{-}$ mitê de Ética da Escola Superior de Educação Física da Universidade Federal de Pelotas, Brasil (protocolo: 4.676.277).

O IFSul conta com 14 Câmpus e a Reitoria em 13 cidades do estado do Rio Grande do Sul (Pelotas, Bagé, Camaquã, Charqueadas, Gravataí, Jaguarão, Lajeado, Novo Hamburgo, Santana do Livramento, Sapiranga, Sapucaia do Sul e Venâncio Aires) - Figura 1. A coleta foi realizada com o intuito de alcançar toda a comunidade do IFSul que possuí, aproximadamente, 21.939 estudantes (sendo 13.311 presenciais e 8.628 a distância), 1.074 servidores docentes (975 professores efetivos e 99 professores substitutos) e 811 técnicos administrativos (Tabela 1).

Inicialmente, o estudo foi pensado com proposta de coleta no Câmpus Pelotas, após foi apresentado à gestão do IFSul e adotado pela mesma como uma pesquisa Institucional. $\mathrm{O}$ acompanhamento foi realizado através de dois momentos de coleta, compreendendo três períodos de tempo distintos. Dessa maneira, os períodos foram divididos em: Coleta 1 - "Pré distanciamento social" e "Durante distanciamento social" (realizada entre maio e agosto de 2021). As questões do pré distanciamento social se referem ao período anterior a deflagração da pandemia de COVID-19; e Coleta 2 - "Pós retorno das aulas presenciais". A Coleta 2 está prevista para o primeiro semestre de 2022.

A partir deste momento, foram iniciadas as ações internas com os setores responsáveis pela Comunicação Social e Departamento de Tecnologia da Informação. Quando todo material informativo e de divulgação estava pronto, assim como os instrumentos já hospedados nas plataformas digitais do Câmpus, foi iniciado o processo de divulgação / comunicação do estudo para comunidade acadêmica ${ }^{19}$.

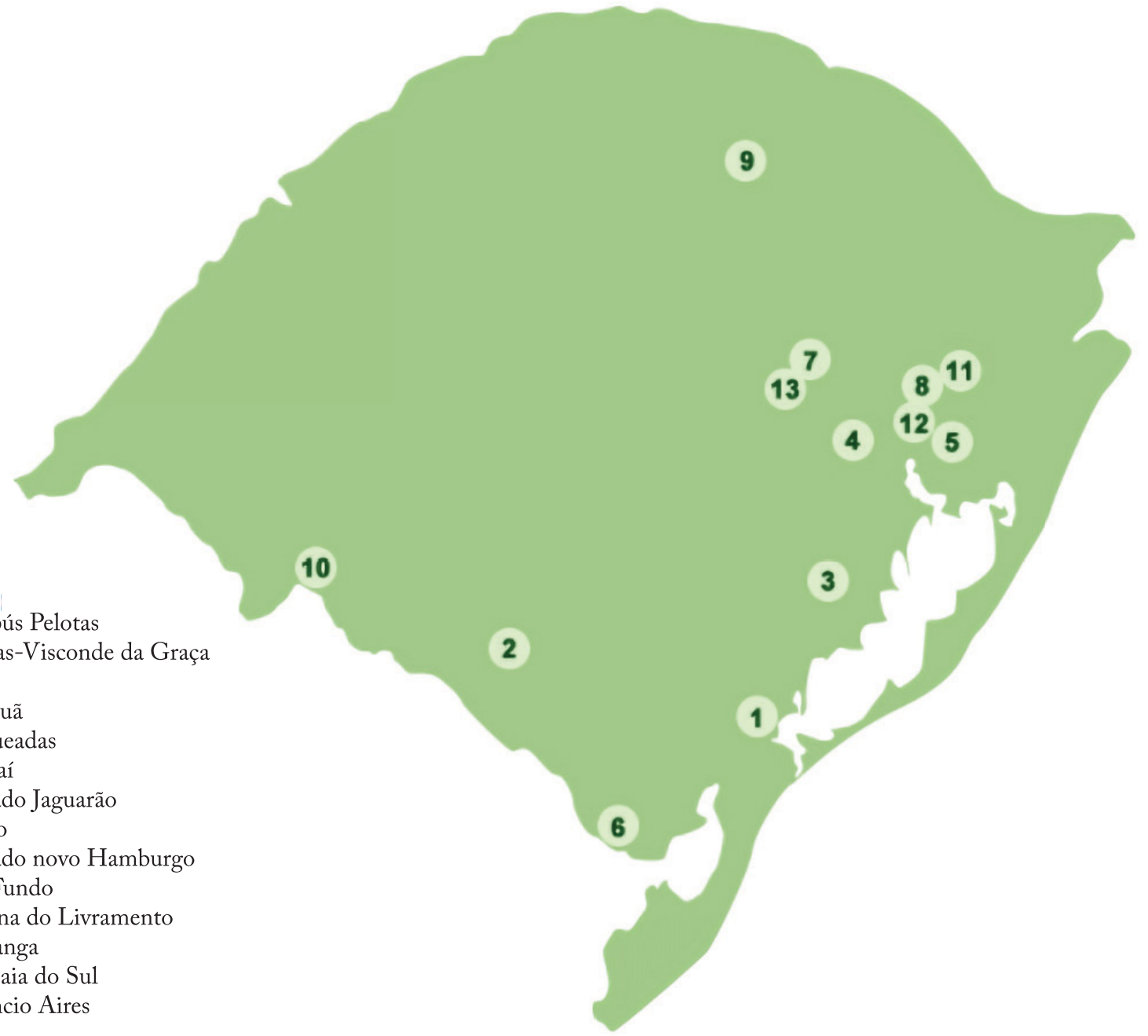

Legenda

1 Reitoria | Campús Pelotas

Campus Pelotas-Visconde da Graça

2 Campus Bagé

3 Campus Camaquã

4 Campus Charqueadas

5 Campus Gravataí

6 Campus Avançado Jaguarão

7 Campus Lajeado

8 Campus Avançado novo Hamburgo

9 Campus Passo Fundo

10 Campus Santana do Livramento

11 Campus Sapiranga

12 Campus Sapucaia do Sul

13 Campus Venâncio Aires

Figura 1 - Mapa do Estado do Rio Grande do Sul com os Câmpus e a Reitoria do Instituto Federal Sul-rio-grandense.

Fonte: Imagem retirada do site oficial do Instituto Federal Sul-Rio-Grandense. 
Tabela 1 - Estimativa do número de estudantes, servidores e técnicos administrativos por Câmpus do Instituto Federal Sul-rio-grandense.

\begin{tabular}{lccc}
\hline Câmpus & Estudantes & Professores & $\begin{array}{c}\text { Técnicos } \\
\text { Administrativos }\end{array}$ \\
\hline Pelotas & 5.545 & 391 & 206 \\
Visconde da Graça & 3.544 & 127 & 79 \\
Sapucaia do Sul & 1.523 & 89 & 54 \\
Charqueadas & 950 & 69 & 39 \\
Passo Fundo & 1.163 & 68 & 46 \\
Camaquã & 1.221 & 53 & 29 \\
Bagé & 464 & 48 & 29 \\
Jaguarão & 321 & 24 & 11 \\
Santana do Livramento & 2.037 & 38 & 26 \\
Novo Hamburgo & 3.039 & 25 & 15 \\
Sapiranga & 477 & 34 & 23 \\
Venâncio Aires & 663 & 54 & 30 \\
Lajeado & 538 & 27 & 16 \\
Gravataí & 454 & 26 & 17 \\
Reitoria & -- & 1 & 191 \\
Total & 21.939 & 1.074 & 811 \\
\hline
\end{tabular}

Fonte: Dados fornecidos pela diretoria das instituições.

Foram criadas comissões locais nos Câmpus, sendo essas responsáveis por auxiliar na coleta dos dados e tendo o grupo de pesquisadores principal como comissão central. O Quadro 1 apresenta o cronograma da Coleta 1 nos diferentes Câmpus.

Para alcançar a população alvo foram utilizadas quatro etapas de divulgação da pesquisa:

- 1a etapa de divulgação da pesquisa: disponibilização do link do instrumento na página inicial do Câmpus e da plataforma digital de acesso as aulas remotas, com utilização de recurso de uma mensagem adicional de "Pop-up", que só era removida com "ok" do usuário. O link também foi inserido em duas plataformas de acompanhamento de frequência e notas.

- 2a etapa de divulgação da pesquisa: Estudantes consistia na disponibilização do link por professores parceiros e de tempo para preenchimento do instrumento em período de aula. Servidores - realizada através do encaminhamento de uma mensagem de e-mail via comunicação social do IFSul, com o link do instrumento expresso diretamente no corpo do texto.

- $3^{\text {a }}$ etapa de divulgação: utilização das mídias sociais (WhatsApp, Instagram e Facebook) para incentivo a participação na pesquisa de estudantes e servidores.

- 4a etapa de divulgação da pesquisa: encaminhamento dos links em mensagens padrões nos grupos de WhatsApp de turmas e setores de servidores, facilitando o acesso ao instrumento. Essa via foi adotada como último recurso, para evitar a circulação do link entre pessoas não vinculadas ao IFSul.

O período de coleta foi de duas a quatro semanas, de acordo com o número de alunos e servidores de cada Câmpus. Cada etapa de divulgação da pesquisa foi se agregando a etapa anterior, acabando juntas, ao final da coleta. Quanto a duração de cada etapa de divulgação da pesquisa, foi utilizado $1 / 4$ do tempo de coleta previsto para cada Câmpus. A coleta respeitou os diferentes calendários letivos das aulas remotas, acontecendo sempre durante o período letivo.

A Coleta 2 compreenderá o período "pós retorno das aulas presenciais" e terá como marco o retorno de todas às aulas presenciais no IFSul. Os procedimentos de coleta de dados, a sequência e duração das etapas de divulgação da pesquisa serão semelhantes ao momento de Coleta 1, e terão início, em todos os 14 Câmpus e Reitoria, num prazo de 45 a 60 dias após o retorno das aulas presenciais.

Através da plataforma QuestionPro, foram desenvolvidos dois instrumentos online (um para servidores e outro para estudantes), autopreenchidos, utilizados para coleta de dados das variáveis relacionadas a prática de atividades físicas, comportamento sedentário, saúde mental, sono, alimentação, fumo, consumo de bebidas alcóolicas, além de dados sociodemográficos e informações relacionadas a COVID-19.

Dois estudos piloto foram realizados em uma turma de formandos (que não fez parte da coleta de dados) de um dos Câmpus do IFSul e com professores de uma escola pública, para que se pudesse testar o entendimento das questões, o tempo de resposta e as falhas no instrumento. $\mathrm{O}$ tempo médio para completar o instrumento foi de 11 minutos e as respostas serviram para adequação do instrumento final.

Ao acessar o link do questionário o estudante/ servidor tinha uma breve apresentação da pesquisa e era encaminhado, se menor de idade, inicialmente ao Termo de Assentimento Livre e Esclarecido (TALE) e logo após, ao Termo de Consentimento Livre e Esclarecido (TCLE), solicitando a anuência de um responsável. No caso de estudante maior de idade ou servidor, eram encaminhados diretamente ao TCLE. Ao consentir, o participante era encaminhado para as questões do formulário. Todos os cuidados éticos para pesquisa em ambiente virtual foram tomados ${ }^{20}$. 
Os momentos em que as variáveis foram/serão coletadas estão descritos no quadro 2. O instrumento conta com informações institucionais referentes a jornadas de estudo e trabalho. Além disso, há variáveis sociodemográficas como idade (em anos completos), gênero (mulher cisgênero, homem cisgênero, mulher transexual, homem transexual, não binário e outro), cor da pele (branco, preto, indígena, pardo, outro), estado civil (solteiro, namorando, casado / morando com companheira, viúvo e separado) e renda familiar (menos que 1 salário mínimo, de 1 até 3 salários mínimos, de 3 até 5 salários mínimos, de 5 até 10 salários mínimos e mais de 10 salários mínimos). Peso e estatura autorreferidos serão utilizados para o cálculo do índice de massa corporal através da fórmula peso/estatura ${ }^{2}$.
A atividade física foi coletada da seguinte forma: $\mathrm{Na}$ Coleta 1 foi mensurada a prática de atividade física antes e durante o distanciamento social, através de duas questões similares. Antes do distanciamento social: "ANTES da Pandemia, você praticava atividade física de forma regular no seu TEMPO LIVRE, como caminhar, andar de bicicleta, praticar algum esporte, etc?" Se sim, a frequência semanal, o tempo médio por dia e o tipo de atividade foram coletados ${ }^{21}$. Da mesma forma, durante o distanciamento social, as questões sobre atividade física tiveram o mesmo formato, incluindo uma questão sobre a intensidade dessa atividade. $\mathrm{Na}$ Coleta 2, será utilizada a seguinte pergunta sobre a prática de atividade física "ATUALMENTE, você pratica atividade física de forma regular no seu TEMPO

Quadro 1 - Período de realização da Coleta 1 (pré distanciamento social e durante o distanciamento social) em cada um dos Câmpus e na Reitoria do Instituto Federal Sul-rio-grandense.

\begin{tabular}{|c|c|c|c|c|c|c|c|}
\hline \multirow{2}{*}{ Câmpus } & \multicolumn{7}{|c|}{ Período de coleta (entre maio e agosto de 2021) } \\
\hline & $11 / 05$ a $13 / 06$ & 12 a $18 / 07$ & 19 a $25 / 07$ & $26 / 07$ a $01 / 08$ & 02 a 08/08 & 09 a $15 / 08$ & 16 a $22 / 08$ \\
\hline \multicolumn{8}{|c|}{ Visconde da Graça } \\
\hline \multicolumn{8}{|l|}{ Pelotas } \\
\hline \multicolumn{8}{|l|}{ Sapucaia } \\
\hline \multicolumn{8}{|c|}{ Charqueadas } \\
\hline \multicolumn{8}{|c|}{ Passo Fundo } \\
\hline \multicolumn{8}{|l|}{ Camaquã } \\
\hline \multicolumn{8}{|l|}{ Bagé } \\
\hline \multicolumn{8}{|l|}{ Jaguarão } \\
\hline \multicolumn{8}{|c|}{ Santana do Livramento } \\
\hline \multicolumn{8}{|c|}{ Novo Hamburgo } \\
\hline \multicolumn{8}{|l|}{ Sapiranga } \\
\hline \multicolumn{8}{|l|}{ Venâncio } \\
\hline \multicolumn{8}{|l|}{ Lajeado } \\
\hline \multicolumn{8}{|l|}{ Gravataí } \\
\hline Reitoria & & & & & & & \\
\hline
\end{tabular}

Quadro 2 - Variáveis coletadas nos diferentes momentos durante o acompanhamento.

\begin{tabular}{|lccc|}
\hline \multicolumn{1}{c}{ Variáveis } & \multicolumn{2}{c|}{ Coleta 1 } & \\
\cline { 2 - 4 } & Pré distanciamento social & Durante o distanciamento social & Pós retorno das aulas presenciais \\
\hline Institucionais & & $\mathrm{X}$ & $\mathrm{X}$ \\
Sociodemográficas & & $\mathrm{X}$ & $\mathrm{X}$ \\
Peso/altura/IMC & $\mathrm{X}$ & $\mathrm{X}$ & $\mathrm{X}$ \\
Atividade Física & $\mathrm{X}$ & $\mathrm{X}$ & $\mathrm{X}$ \\
Comportamento sedentário & $\mathrm{X}$ & $\mathrm{X}$ & $\mathrm{X}$ \\
Saúde Mental & & $\mathrm{X}$ & $\mathrm{X}$ \\
Autopercepção de saúde & $\mathrm{X}$ & $\mathrm{X}$ & $\mathrm{X}$ \\
Comportamentais* & $\mathrm{X}$ & $\mathrm{X}$ & $\mathrm{X}$ \\
COVID-19 & & $\mathrm{X}$ & $\mathrm{X}$ \\
\hline
\end{tabular}

* Sono/Alimentação/Tabaco/Alcool. 
LIVRE, como caminhar, andar de bicicleta, praticar algum esporte, etc?" Se sim, serão coletadas a frequência semanal, o tempo médio por dia e o tipo de atividade. Estudos anteriores utilizaram questões semelhantes para mensurar a pratica de atividade física ${ }^{5,21,22}$. Os participantes serão classificados quanto a prática de atividade física de acordo com as recomendações da Organização Mundial da Saúde ${ }^{23}$.

$\mathrm{Na}$ Coleta 1, para os estudantes, foi questionado sobre a participação em atividades físicas/ esportivas oferecidas pelo IFSul no período "pré distanciamento social". Aqueles que responderam sim, foram perguntados sobre qual modalidade participaram e o tempo de participação em cada uma delas.

O comportamento sedentário foi perguntado da seguinte forma na Coleta 1: uma questão sobre autopercepção de mudança de comportamento sedentário no lazer e outra no trabalho/estudo, comparando o período atual com o período anterior ao distanciamento social. Além disso, foram questionados os tempos de comportamento sedentário através de duas questões, sendo uma para lazer e outra para trabalho/estudo: "Atualmente, quanto tempo (em minutos) por dia, você assiste televisão ou fica na frente do computador ou celular por (lazer) ou (trabalho/ estudo)?”. Na Coleta 2, serão repetidas as questões referentes aos tempos de comportamento sedentário no lazer e trabalho/estudo.

O desfecho saúde mental esteve presente na Coleta 1 (referente apenas ao período "durante o distanciamento social”) e estará na Coleta 2, sendo mensurado através da Hospital Anxiety and Depression Scale (HADS), que identifica sintomas de depressão e ansiedade. Essa é uma escala simples e confiável ${ }^{24}$ que conta com 14 afirmações, sendo 7 delas relacionadas a depressão e 7 a ansiedade. Cada afirmação possui uma escala de Likert variando de 0 a 3 pontos. $\mathrm{O}$ escore total para cada domínio varia de 0 a 21 pontos. Os participantes que apresentarem somatório abaixo de 7 serão classificados como "sem sintomas", entre 8 e 10 serão consideradas "sintomas leves", entre 11 e 14 com "sintomas moderados" e acima de 15 com "sintomas severos" de depressão e/ou ansiedade ${ }^{25}$.

Questões sobre autopercepção de mudança relacionados ao sono, consumo de alimentos saudáveis e não saudáveis, tabagismo e consumo de bebidas alcóolicas fazem parte do questionário, sendo aplicadas nos diferentes períodos de coleta. Para cada uma dessas variáveis comportamentais foi utilizada uma variante da pergunta: "Comparado ao período anterior a pandemia, em relação a [variável de interesse], hoje você acha que está", com alternativas de resposta a manutenção, o aumento ou a diminuição do comportamento. $\mathrm{Na}$ Coleta 1 foi questionada a autopercepção de saúde "durante o distanciamento", assim como, a mudança dessa autopercepção em comparação ao período "pré distanciamento social”. $\mathrm{Na}$ Coleta 2 será questionada a autopercepção de saúde no momento da coleta.

Informações referentes a vacinação (se já se vacinou, se pretende se vacinar e quantas doses da vacina tomou); diagnóstico de COVID-19, pessoal e de coabitantes ( $\operatorname{sim} /$ não); nível de preocupação com a COVID-19 (muito preocupado; preocupado; pouco preocupado, não está preocupado); cumprimento de medidas de distanciamento social (totalmente isolado; saindo apenas para atividades essenciais; saindo, usando máscara e álcool gel; saindo normalmente) e falecimento de pessoa próxima por COVID-19 ( $\mathrm{sim} /$ não), serão utilizadas em ambas coletas ${ }^{22}$.

Os dados serão exportados do QuestionPro para o Microsoft ${ }^{\circledR}$ Excel e transferidos ao Stata 13.1, onde a análise será realizada. A amostra será caracterizada através de estatística descritiva (média e desvio padrão ou prevalência e intervalo de confiança de 95\%). Para testar a normalidade dos dados será utilizado o teste de Shapiro Wilk. Para desfechos categóricos será utilizado o teste Qui-quadrado e para desfechos contínuos será utilizado ANOVA. Regressão Linear Múltipla para desfechos contínuos e regressão de Poisson para desfechos categóricos serão utilizadas para as análises ajustadas. Generalized Estimating Equations (GEE) ou ANOVA para medidas repetidas serão utilizadas para comparações entre os momentos (Pré, durante e pós distanciamento social), o teste post hoc de Bonferroni será utilizado quando necessário.

\section{Discussão}

Ao nosso conhecimento, esse será o primeiro estudo longitudinal realizado no ambiente escolar que objetiva verificar o impacto do distanciamento social em variáveis de saúde de estudantes e servidores de uma instituição pública e com abrangência estadual. Estudos anteriores verificaram que o distanciamento social afeta negativamente, principalmente, a saúde mental da populaçãa ${ }^{13,26}$ e a prática de atividade física ${ }^{27}$. Especificamente, no Rio Grande do Sul, um estudo longitudinal mostrou que durante o distanciamento social houve uma piora na saúde mental da população gaú- 
cha $^{5}$. Deste modo, no ambiente escolar, a diminuição das atividades realizadas em ambiente fechado ${ }^{12}$, ao ar livre e a redução na interação social entre os escolares e servidores durante o período de ensino remoto, podem acarretar graves consequências na saúde e no desenvolvimento, principalmente, de crianças e adolescentes ${ }^{6}$.

O presente estudo apresenta algumas limitações, como o instrumento ser auto aplicado, via internet. Para facilitar a adesão ao estudo, os autores optaram por não utilizar um questionário muito longo, dificultando assim, a utilização de instrumentos validados. Outra limitação do estudo é o viés de memória, tendo em vista que, na Coleta 1 (realizada entre maio e agosto de 2021) os estudantes responderam informações referentes ao período anterior ao distanciamento social.

Para enfrentamento dos problemas encontrados ao longo da pesquisa, serão sugeridas ações pontuais para docente, TAEs e discentes, como por exemplo, palestras e lives com divulgação dos resultados da pesquisa para a comunidade, também podem ser traçadas estratégias para redução do comportamento sedentário e aumento dos níveis de atividade física, através de parcerias entre as direções dos Câmpus e os professores de educação física.

Como hipótese para o presente estudo, tem-se que os níveis de atividade física serão menores tanto em alunos quanto em servidores durante o distanciamento social. Ainda, durante o período de distanciamento social, espera-se que os indicadores de saúde apresentem piores resultados quando comparados aos períodos de aulas presenciais, impulsionados pela redução da prática de atividades físicas tanto para estudantes quanto servidores do IFSul. Quando comparados os momentos pré e pós distanciamento social, espera-se que os níveis de atividades físicas e os indicadores de saúde apresentem valores semelhantes em ambas as populações investigadas.

Dessa forma, entender como o distanciamento social afetou a saúde de estudantes e servidores pode ser uma ferramenta importante para os gestores criarem políticas de retorno das aulas, bem como, estabelecer um período de acompanhamento após o retorno das atividades para criação de estratégias de promoção de saúde, nas variáveis mais afetadas, entre estudantes e servidores.

\section{Conflito de interesse}

Os autores declaram não haver conflito de interesse.

\section{Contribuição dos autores}

Häfele CA, Kremer MM e Borges TT participaram da concep- ção inicial do manuscrito e da revisão crítica do manuscrito. Guimarães IF e Foster FM participaram da confecção de figuras e tabelas e da revisão crítica do texto. Cunha GB, Cunha GOK, Reichert $\mathrm{FF}$ e Bacchieri $\mathrm{G}$ realizaram a redação do manuscrito e revisão crítica do conteúdo.

\section{Agradecimentos}

Os autores agradecem a Reitoria do Instituto Federal Sul-rio-grandense e a direção de cada um dos 14 Campi por viabilizar os procedimentos de coleta de dados.

\section{Referências}

1. Brasil. Ministério da Saúde do Brasil. Painel do Coronavirus - Brasil. 2021; Disponível em: https://covid.saude.gov.br/ [2021 junho].

2. WHO. Director-General's opening remarks at the media briefing on COVID-19. World Health Organization. 2020; Disponível em: https:/www.who.int/director-general/ speeches/detail/who-director-general-s-opening-remarksat-the-media-briefing-on-covid-19---11-march-2020 [2021 setembro].

3. Pires RRC. Os efeitos sobre grupos sociais e territórios vulnerabilizados das medidas de enfrentamento à crise sanitária da covid-19: propostas para o aperfeiçoamento da ação pública: Nota Técnica. Instituto de Pesquisa Econômica Aplicada (Ipea). 2020;33:1-11.

4. UNESCO calls for investimento in quality physical education to support COVID-19 recovery. 2021; Disponível em: https://en.unesco.org/news/unesco-calls-investmentquality-physical-education-support-covid-19-recovery [2021 setembro].

5. Feter N, Caputo EL, Doring IR, Leite JS, Cassuriaga J, Reichert FF, et al. Sharp increase in depression and anxiety among Brazilian adults during the COVID-19 pandemic: findings from the PAMPA cohort. Public Health. 2021;190:101-7.

6. Bergmann GG, Cunha GB, Cunha GOK, Cruz JHB, Silva LR, Ferreira GD, et al. Changes in body weight and health behaviors of overweight children during the COVID-19 pandemic. Rev Bras Ativ Fís Saúde. 2020;25:e0153.

7. Pietrobelli A, Pecoraro L, Ferruzzi L, Heo M, Faith M, Zoller T, et al. Effects of COVID-19 Lockdown on Lifestyle Behaviors in Children with Obesity Living in Verona, Italy: A Longitudinal Study. Obesity. 2020;28(8):1382-85.

8. Machado RB, Fonseca DG, Medeiros FM, Fernandes N. Educação Física escolar em tempos de distanciamento social: panorama, desafios e enfrentamentos curriculares. Movimento. 2020;26:e26081.

9. Brooks SK, Webster RK, Smith LE, Woodland L, Wessely S, Greenberg N, et al. The psychological impact of quarantine and how to reduce it: rapid review of the evidence. Lancet. 2020;395(10227):912-20.

10. Silva LCB, Novaes CRMN, Lima Júnior RA, Giudicelli BB, Cunha Júnior AT, Tenório MCM, et al. Sleep, sedentary behavior and physical activity: changes on children's routine during the COVID-19. Rev Bras Ativ Fís Saúde. 2020;25:e0143.

11. Cunha GOK, Cunha GB, Hartwig TW, Bergmann GG. Association between changes in physical activity levels and depressive symptoms in high school students during the COVID-19 pandemic. J Mov Heal. 2022;19(1):1-10. 
12. Brito LMS, Boguszewski MCS, Souza MTR, Martins F, Mota J, Leite N. Indoor physical activities, eating and sleeping habits among school adolescents during COVID-19 pandemic. Rev Bras Ativ Fís Saúde. 2020;25:e0117.

13. Pierce M, Hope H, Ford T, Hatch S, Hotopf M, John A, et al. Mental health before and during the COVID-19 pandemic: a longitudinal probability sample survey of the UK population. Lancet Psychiat. 2020;7(10)0366:883-92.

14. Solon Júnior LJF, Fortes LS, Barbosa BT, Feitosa Júnior JVA, Ribeiro CHT, Neto LVS. Home-based exercise during confinement in COVID-19 pandemic and mental health in adults: a cross sectional comparative study. Rev Bras Ativ Fís Saúde. 2020;25:e0120.

15. Godoi M, Kawashima L, Gomes LA, Caneva C. O ensino remoto durante a pandemia de COVID-19: desafios, aprendizagens e expectativas dos professores universitários de educação física. Res Soc Dev. 2020;9(10):1-19.

16. Santos, GMRF, Silva ME, Belmonte BR.COVID-19: Ensino remoto emergencial e saúde mental de docentes universitários. Rev Bras Saúde Mater Infant. 2021;21(Supl.1):S245-S251.

17. Casagrande M, Favieri F, Tambelli R, Forte G. The enemy who sealed the world: effects quarantine due to the COVID-19 on sleep quality, anxiety, and psychological distress in the Italian population. Sleep Med. 2020;75:12-20.

18. Wang G, Zhang Y, Zhao J, Zhang J, Jiang F. Mitigate the effects of home confinement on children during the COVID-19 outbreak. Lancet. 2020;395(10228):945-7.

19. Brasil. Instituto Federal Sul-riograndense, Câmpus Pelotas. Pesquisa vai avaliar condições de saúde e hábitos de alunos e servidores durante a pandemia.2021; Disponível em: <http:// www.pelotas.ifsul.edu.br/pesquisa-vai-avaliar-condicoesde-saude-e-habitos-de-alunos-e-servidores-durante-apandemia> [2021 dezembro].
20. Brasil. Ministério da Saúde. Orientações para procedimentos em pesquisas com qualquer etapa em ambiente virtual. 2021; Disponível em: <http://conselho.saude.gov.br/ images/comissoes/conep/documentos/CARTAS/Carta_ Circular_01.2021.pdf>. [2021 dezembro].

21. Milton K, Bull FC, Bauman A. Reliability and validity testing of a single-item physical activity measure. Br J Sports Med. 2011;45(3):203e8.

22. Feter N, Caputo EL, Doring IR, Leite JS, Cassuriaga J, Reichert FF, et al. Longitudinal study about low back pain, mental health, and access to healthcare system during COVID-19 pandemic: protocol of an ambispective cohort Short title: PAMPA cohort: study protocol. medRxiv. Cold Spring Harbor Laboratory Press. 2020;Jul:1e11.

23. WHO. Global recommendations on Physical Activity for Health - World Health Organization. Geneva; 2010.

24. Bjelland I, Dahl AA, Haug TT, Neckelmann D. The validity of the Hospital Anxiety and Depression Scale: an updated literature review. J Psychosom Res. 2002;52(2):69-77.

25. Zigmond AS, Snaith RP. The hospital anxiety and depression scale. Acta Psychiatr Scand. 1983;67(6):361-70.

26. Pieh C., Budimir S., Probst T. The effect of age, gender, income, work, and physical activity on mental health during coronavirus disease (COVID-19) lockdown in Austria. J Psychosom Res. 2020;136:110186.

27. Costa CLA, Costa TM, Barbosa Filho VC, Siqueira RCL. Influência do distanciamento social no nível de atividade física durante a pandemia do COVID-19. Rev Bras Ativ Fís Saúde. 2020;25:e0123.

Recebido: 26/08/2021

Aprovado: 22/01/2022

\section{Como citar este artigo:}

Häfele CA, Kremer MM, Cunha GB, Cunha GOK, Guimarães IF, Foster FM, Bacchieri G, Reichert FF, Borges TT. Projeto RESPIRE: protocolo de avaliação do impacto da pandemia na saúde da comunidade do IFSul. Rev Bras Ativ Fís Saúde. 2022;27:e0244. DOI: 10.12820/rbafs.27e0244 